\title{
Prevalence of Obesity among Female Patients with Rosacea
}

\author{
Saima Rahman ${ }^{1}$, Nisaruddin ${ }^{2}$, Aafaque Ahmad ${ }^{2}$
}

\begin{abstract}
Background: Rosacea is a chronic skin (mainly facial skin) disease of unclear origin. Epidemiological data are scarce and controversial, with reported prevalence ranging from $0.09 \%$ to $22 \%$. To our knowledge, prevalence of obesity among female patients with rosacea has not been understood.

Objective: We sought to investigate the relation between rosacea in female patients with prevalence of obesity.

Material \& Methods: Between January and June 2018, a study including 230 female rosacea patients were observed to understand the prevalence of obesity in these patients. Demographic data, clinical features of rosacea patients, anthropometric measures and BMI were recorded.

Results: Two hundred and thirty female participants enrolled and completed the survey. Among the total, 188 participants were having Erythematotelangiectatic rosacea variant and 42 were classified as papulopustular rosacea. Of the total 26 were having $\mathrm{BMI}<21$ while 7 had BMI $>35$. The maximum participants 97 had BMI ranging 25-29.9.

Conclusion: There is significant relationship between obesity and rosacea. This is a window to further research to help to conclude if addressing obesity may help improve rosacea.
\end{abstract}

Keywords: Obesity, rosacea, BMI.

This article may be cited as: Rahman S, Nisaruddin, Ahmad A.

Prevalence of Obesity among Female Patients with Rosacea. J Saidu Med Coll Swat 2020;10(2):147-149

\section{INTRODUCTION}

Rosacea is a chronic inflammatory skin condition particularly affecting the face but may involve the eyes. ${ }^{1}$ Disease occur as persistent or recurrent episodes of centrofacial erythema and flushing. ${ }^{1-3}$ Rosacea most often occurs in people aged 20-50 years ${ }^{4}$ and it has 4 subtypes out of which the most common one is Erythemotelangiestatic (ETR). ETR is typified by persistent centrofacial erythema and flushing with the presence or absence of telangiectases. $^{8}$ The second most common subtype of rosacea is papulopustular rosacea (PPR), which consists of persistent centrofacial erythema along with transient, central facial papules and/or pustules. ${ }^{5,6}$ The remaining two types are phymatous and ocular. Besides these subtypes, other variants are granulomatous rosacea, periorificial dermatitis, pyoderma faciale and steroid induced rosacea. ${ }^{1,7,8}$

The pathophysiology of rosacea is unclear, and epidemiologic risk factors for diverse events in rosacea are poorly understood. However, data suggest that Demodix folliculorum and other organisms along with UV light and genetic predisposition leads to abnormally functioning

\footnotetext{
1.Department of Dermatology, Saidu Group of Teaching Hospitals Saidu Sharif, Swat.

2.Department of Rehabilitation Medicine Saidu Group of Teaching Hospitals Saidu Sharif Swat.
}

Correspondence: Dr. Nisaruddin

Head of Department Rehab Medicine,

Saidu Group of Teaching Hospital, Swat.

Email: drnisaruddinphysiatrist@gmail.com

Cell No: 03314980820 innate immune detection and response system, which induces activation of inflammatory cascades. ${ }^{5,6}$ Vasodilation in rosacea is due to an outcome of neurovascular dysregulation. ${ }^{10}$ In addition to the unpleasant symptomatic effects of rosacea, its substantial psychosocial impact leads to substantial effects on quality of life (QoL).

According to the 2016 World Health Organization (WHO) statistics, $22.7 \%$ of female population of Pakistan is overweight and $6.4 \%$ of female population is obese. ${ }^{9}$ Obesity leads to multiple adverse health effects, including decreased longevity, diabetes mellitus, and cancer, ${ }^{10}$ and has also been associated with several inflammatory skin diseases, such as psoriasis, ${ }^{11}$ acne, ${ }^{12}$ and atopic dermatitis. ${ }^{15}$ Further, obesity affects cutaneous vasculature, sensation, and temperature regulation. ${ }^{13,}{ }^{14}$ We therefore hypothesized that obesity might be associated with a risk for rosacea in female patients, because of its hallmark inflammatory, vascular, and skin sensitivity as clinical features.

Associations between obesity and rosacea have been inconsistent in previous epidemiologic studies. ${ }^{15-18}$ To our knowledge, no research study has been conducted to understand the prevalence of obesity among female patients with rosacea.

In this study, we investigated the association between BMI and Rosacea in a total of 230 participants from the Department of Dermatology, Saidu Teaching Hospital, Saidu Sharif Swat, KPK Pakistan. 


\section{MATERIAL AND METHODS}

This study involved 230 female patients aged $=18$ years visiting the Department of Dermatology, Saidu Teaching Hospital, Saidu Sharif Swat, KPK Pakistan. The selection of female patients was by chance as no male patient visited the OPD for consultation. This further emphasized the disease being more common in female patients, again reflecting that female patient Quality of life is more affected. On this basis the title was revisited and female patients with rosacea mentioned. Moreover, the female patients with induced rosacea and other dermatosis were excluded resulting in the reduced sample size. The study was performed between $1^{\text {st }}$ January and $30^{\text {th }}$ June 2018. Inclusion criteria required the female subjects to be of an appropriate age, to have maintained informed written consent for the purpose of medical history taking, and to demonstrate conscious agreement to her participation in the study. All participants were thoroughly evaluated in a stratified clinical interview and a physical examination with special emphasis was placed on excluding other dermatological issues. Special emphasis was made to include those with papulopustular and Erythemotelangiestatic type, because of being major subtypes and being commonest. No patient was received with phymatous type and induced rosacea was excluded purposely.

\section{Statistical Analysis}

All collected data were statistically analyzed using SPSS Version 24. P-values of $<0.05$ were considered to indicate statistical significance.

\section{RESULTS}

Among total of 230 patients who presented with Rosacea to the Skin OPD, 188 (81.74\%) were having Erythemotelangiestatic type and 42
(18.26\%) were having papulopustular rosacea. Only $1 / 230$ patient $(0.53 \%)$ was underweight and had Erythemotelangiestatic type rosacea. Total of $52(22.61 \%)$ were obese, among which 41 $(78.84 \%)$ were having Erythemotelangiestatic Rosacea. Papulopustular type contributed as 11 $(21.16 \%)$ of the total obese with rosacea. Of the total 230 sample size $97(42.17 \%)$ were overweight, of which $79(81.5 \%)$ were of Erythemotelangiestatic type and (18.5\%) were papulopustular. The rest that is $80 / 230$ (67-ET \& 13PP) patients ranged in normal BMI.

\section{DISCUSSION}

We found that obesity was associated with increased risk for rosacea. To our knowledge, this is the first cohort study on the association between obesity and risk for incident rosacea.

The previous literature shows inconsistent findings on the association between obesity and rosacea. Two case-control studies indicated null findings. ${ }^{11,}{ }^{12}$ One based on the General Practice Research Database (Oxford, UK) showed neither high nor low BMI to be associated with an altered risk for rosacea, ${ }^{11}$ and the other small-scale casecontrol study from Turkey indicated no significant difference of BMI between rosacea group and controls. ${ }^{12}$ However, a cross-sectional study in Poland showed a statistically significant relationship between increased $\mathrm{BMI}$ and rosacea, ${ }^{19}$ and another cohort-based survey of twins in the United States showed a positive correlation between BMI and rosacea severity scores. ${ }^{13}$

We showed significantly positive associations between rosacea with raised BMI.

Table 1. Comparison of Rosacea with BMI

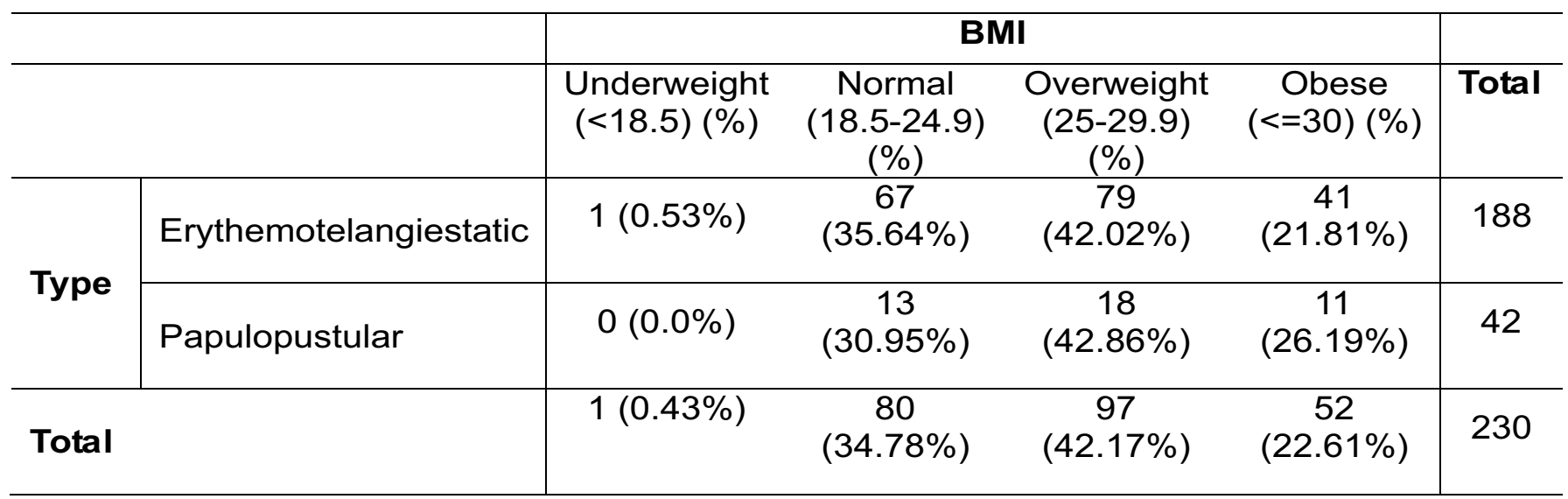


A major strength of our study is the prospectively collected data. Because individuals' obesity can change over time, updating information on obesity ensured that we used the most accurate information for our exposure, thus avoiding misclassification. We acknowledge some limitations, like taking BMI without establishing relationship with Waist Hip circumference or ratio, obese participants might be more likely to see their physician for comorbidities and therefore be incidentally diagnosed with rosacea. On the other hand, obese participants might defer complaints related to relatively minor skin diseases in favor of focusing on more serious comorbidities. Another limitation is the lack of information on rosacea subtypes; different types of rosacea have known etiologic heterogeneity. ${ }^{1,6}$ Third, participants were women, and most were of single race; therefore the compare and contrast to other studies (discussion) was limited. Extrapolation of our findings to men and other races/ethnicities and other variants of rosacea should be approached with caution.

In summary, we provide evidence that obesity might be associated with an increased risk for rosacea. The study contributes to the understanding of rosacea etiology and informs clinical practice related to rosacea associations.

\section{CONCLUSION}

In summary, chronic condition like Rosacea needs to be addressed from all the aspects. Therapies that address the underlying cause is the only means to deal successfully with the unsightly appearance and the bothersome symptoms of rosacea. Again, with appropriate education regarding etiology and treatment is needed for better outcome. This study contributes to the understanding of rosacea etiology and associations and gives an initiative regarding clinical practice related to rosacea associations. In future research we look forward to the expected fact, if treating obesity is going to treat/improve Rosacea?

\section{REFERENCES}

1. Steinhoff M, Schauber J, Leyden JJ. New insights into rosacea pathophysiology: a review of recent findings. Journal of the American Academy of Dermatology. 2013;69(6):S15-S26.

2. Elewski B, Draelos Z, Dreno B, Jansen T, Layton A, Picardo M. Rosaceaglobal diversity and optimized outcome: proposed international consensus from the Rosacea International Expert Group. Journal of the European Academy of Dermatology and Venereology. 2011;25(2):188-200.
3. Okhovat J-P, Armstrong AW. Updates in rosacea: epidemiology, risk factors, and management strategies. Current Dermatology Reports. 2014;3(1):23-8.

4. Gupta A, Chaudhry M. Rosacea and its management: an overview. Journal of the European Academy of Dermatology and Venereology. 2005;19(3):273-85.

5. Wilkin J, Dahl M, Detmar M, Drake L, Feinstein A, Odom R, et al. Standard classification of rosacea: report of the National Rosacea Society Expert Committee on the Classification and Staging of Rosacea. Journal of the American Academy of Dermatology. 2002;46(4):584-7.

6. Del Rosso JQ, Thiboutot D, Gallo R, Webster G, Tanghetti E, Eichenfield $\mathrm{L}$, et al. Consensus recommendations from the American Acne \& Rosacea Society on the management of rosacea, part 1: a status report on the disease state, general measures, and adjunctive skin care. Cutis. 2013;92:234-40.

7. Two AM, Wu W, Gallo RL, Hata TR. Rosacea: part I. Introduction, categorization, histology, pathogenesis, and risk factors. Journal of the American Academy of Dermatology. 2015;72(5):749-58.

8. Crawford GH, Pelle MT, James WD. Rosacea: I. Etiology, pathogenesis, and subtype classification. Journal of the American Academy of Dermatology. 2004;51(3):327-41.

9. World Health Organization 2016 [Available from: ht t p://www. who.int/diabetes/countryprofiles/pak_en.pdf?ua=1.

10. Haslam DW, James WP. Obesity. Lancet (London, England). 2005;366(9492):1197-209.

11. Setty AR, Curhan G, Choi HK. Obesity, waist circumference, weight change, and the risk of psoriasis in women: Nurses' Health Study II. Archives of internal medicine. 2007;167(15):1670-5.

12. Tsai MC, Chen W, Cheng YW, Wang CY, Chen GY, Hsu T-J. Higher body mass index is a significant risk factor for acne formation in schoolchildren. European Journal of Dermatology. 2006;16(3):251-3.

13. Scheinfeld NS. Obesity and dermatology. Clinics in dermatology. 2004;22(4):303-9.

14. Kapiotis S, Holzer G, Schaller G Haumer M, Widhalm $H$, Weghuber $D$, et al. A proinflammatory state is detectable in obese children and is accompanied by functional and morphological vascular changes. Arteriosclerosis, thrombosis, and vascular biology. 2006;26(11):2541-6.

15. Spoendlin J, Voegel J, Jick S, Meier C.A study on the epidemiology of rosacea in the UK. British journal of dermatology. 2012;167(3):598-605.

16. Duman N, Ersoy Evans S, Atakan N. Rosacea and cardiovascular risk factors: a case control study. Journal of the European Academy of Dermatology and Venereology. 2014;28(9):1165-9.

17. Reszke R, Pełka D, Walasek A, Machaj Z, Reich A. Skin disorders in elderly subjects. International journal of dermatology. 2015;54(9):e332-e8.

18. Aldrich N, Gerstenblith M, Fu P, Tuttle MS, Varma P, Gotow E, et al. Genetic vs environmental factors that correlate with rosacea: a cohort-based survey of twins. JAMA dermatology. 2015;151(11):1213-9.

19. Drucker A, Li W, Lin L, Cho E, LiT, Camargo Jr C, et al. Atopic dermatitis (eczema) in US female nurses: lifestyle risk factors and atopic co-morbidities. The British journal of dermatology. 2016;174(6):1395. 